\title{
A Polyoxometalate-Assisted Electrochemical Method for Silicon Nanostructures Preparation: From Quantum Dots to Nanowires
}

\section{(Supporting information)}

Zhenhui Kang, ${ }^{a, b}$ Chihim A. Tsang, ${ }^{a, b}$ Zhendong Zhang, ${ }^{a, b}$ Mingliang Zhang, ${ }^{a, b}$ Ningbew Wong, ${ }^{*}{ }^{a, b}$ J. Antonio Zapien, ${ }^{b . c}$ Yueyue Shan, ${ }^{b, c}$ Shuittong Lee* ${ }^{*, c}$

${ }^{a}$ Department of Biology and Chemistry, City University of Hong Kong, Hong Kong SAR, China; E-mail: bhnbwong@cityu.edu.hk

${ }^{\mathrm{b}}$ Centre of Super-Diamond and Advanced Films (COSDAF), City University of Hong Kong, Hong Kong, P. R. China

${ }^{c}$ Department of Physics and Materials Science, City University of Hong Kong, Hong Kong SAR, China; E-mail: apannale@cityu.edu.hk

\section{Experimental details.}

All the chemicals were purchased from Sigma-Aldrich. Briefly, n-type (boron-doped) and p-type (phosphorus-doped) Si chips were cleaned in 20\% hydrofluoric acid (HF) for 5 min first to remove surface oxides and impurities. The electrolyte of the electrochemical etching process was prepared by mixing $60 \mathrm{~mL}$ of ethanol / $\mathrm{HF}(3: 2)$ with a suitable amount $(5-10 \mathrm{~mL})$ of $\mathrm{H}_{2} \mathrm{O}_{2}$ (hydrogen peroxide $30 \%$ ) and POM as the catalyst. POM in ethanol solution $\left(10^{-2}-10^{-3} \mathrm{M}\right)$ was used. The composition of HPOM catalyst was $\mathrm{H}_{2} \mathrm{O}_{2}$ /POM: 97:3 to $90: 10$ (V: V). The electrochemical system composed of graphite as anode and $\mathrm{Si}$ wafer (n-type or p-type, 8-30 $\Omega$ resistivity, about $1 \mathrm{~cm}$ in width, and the total reaction area $0.5-1 \mathrm{~cm}^{2}$ ) as cathode. Graphite and Si wafer were put into electrolyte and connected to the DC power supply by connecting wires. There was a layer of n-hexane covering the electrolyte solution. The current intensity ranged $2-50 \mathrm{~mA} / \mathrm{cm}^{2}$ and the whole etching process was performed for several hours. 


\section{Details of characterization and Results.}

SiQDs and silicon nanostructures were characterized by SEM, TEM, FTIR and PL. The SEM image of SiQDs and other silicon nanostructures sample were obtained by Philips XL30 X-ray source attached to the SEM system. The TEM image of SiQDs was obtained with a FEI/Philips Techal 12 BioTWIN TEM, while HR-TEM image was obtained with a CM200 FEG transmission electron microscope. The FTIR spectrum of SiQDs was obtained with a Nicolet 360 spectrometer. The PL study of SiQDs (coated on quartz plate) was carried out on a PERKIN ELMER Luminescence Spectrometer LS50B. The morphology of silicon nanostructures was characterized by SEM and TEM. Energy disperses spectrum (EDS) results show the compositions of the products are pure silicon.

Figure $\mathrm{S} 1$ shows the TEM image of SiQDs with different diameters: $\sim 1, \sim 2, \sim 3$, and $\sim 4 \mathrm{~nm}$.

Figure S2 shows the size distributions of SiQDs as determined from TEM images.

Figure $\mathrm{S} 3$ shows the excitation spectra of 1, 2, 3, and $4 \mathrm{~nm}$ SiQDs.

Figure S4 shows the typical IR spectrum of SiQDs.

Figure S5 shows the TEM image of SiNPs with diameters about 30-50 nm.

Figure S6 shows the SEM image of silicon nanoparticles-micropore structures.

Figure S7 and S8 show the SEM images of silicon nanorods-microporous structures.

Figure S9 shows the SEM image of nanowires-microporous structures. Figure S10 shows the EDS spectrum of Si nanowires-microporous structures.

Figure S11 and S12 show the SEM image of the products are the micro holes nanowires-microporous when using $\mathrm{H}_{2} \mathrm{O}_{2}$ as additive.

Figure S13-S15 show the SEM image of the products at high current intensity $\left(>50 \mathrm{~mA} / \mathrm{cm}^{2}\right)$.

Figure S16 shows the scheme for the present POMs-assisted electrochemical etching reaction. 


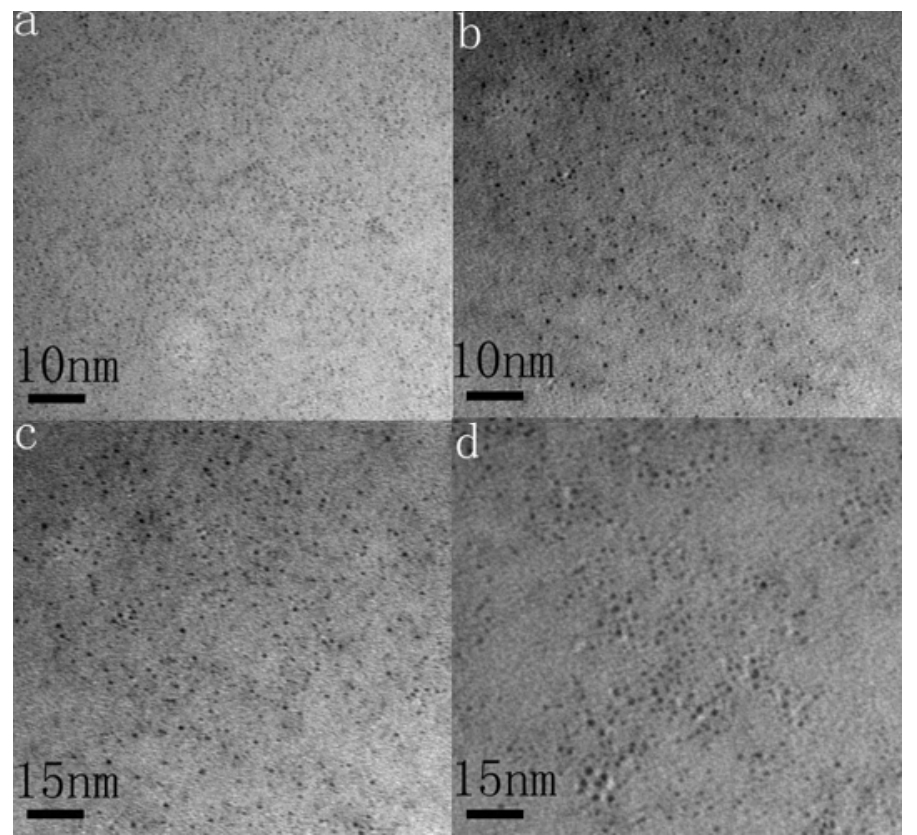

Figure S1 TEM images of SiQDs with different diameters: $\sim 1, \sim 2, \sim 3$, and $\sim 4 \mathrm{~nm}$ for a, b, c, and d, respectively. HPOM is the catalyst. Current density is less than $20 \mathrm{~mA} / \mathrm{cm}^{2}$.
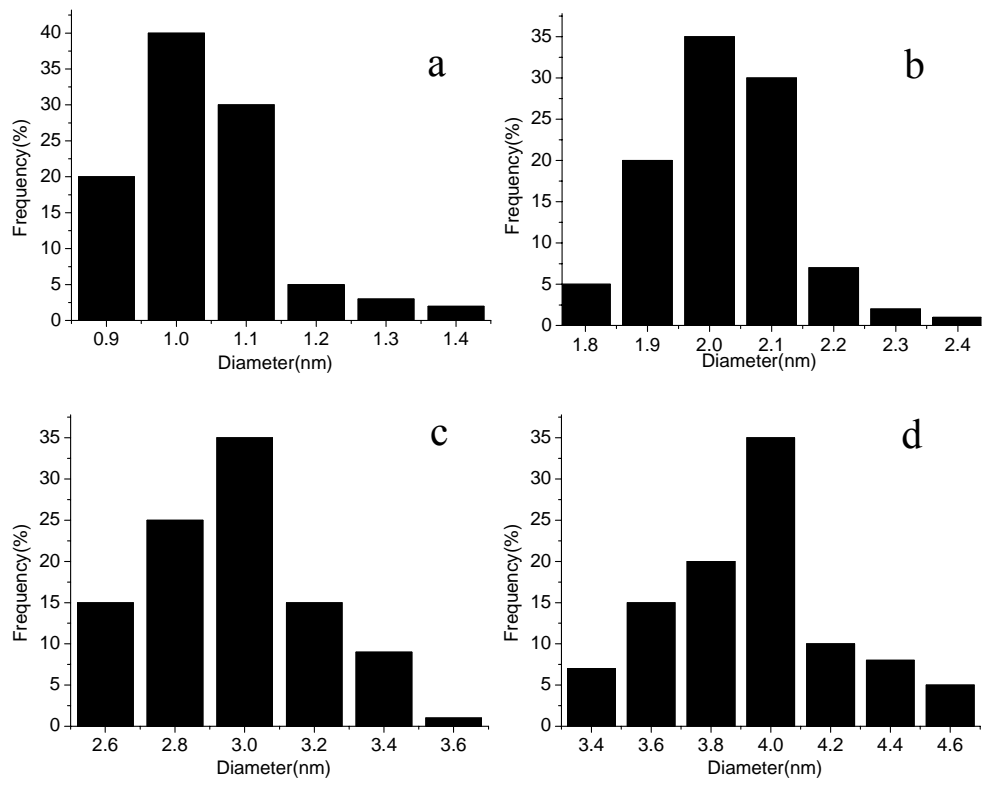

Figure S2 SiQDs size distributions obtained from TEM images; $a, b, c$, and $d$ for $\sim 1, \sim 2, \sim 3$, and $\sim 4 \mathrm{~nm}$, respectively. HPOM is the catalyst. Current density is less than $20 \mathrm{~mA} / \mathrm{cm}^{2}$. 

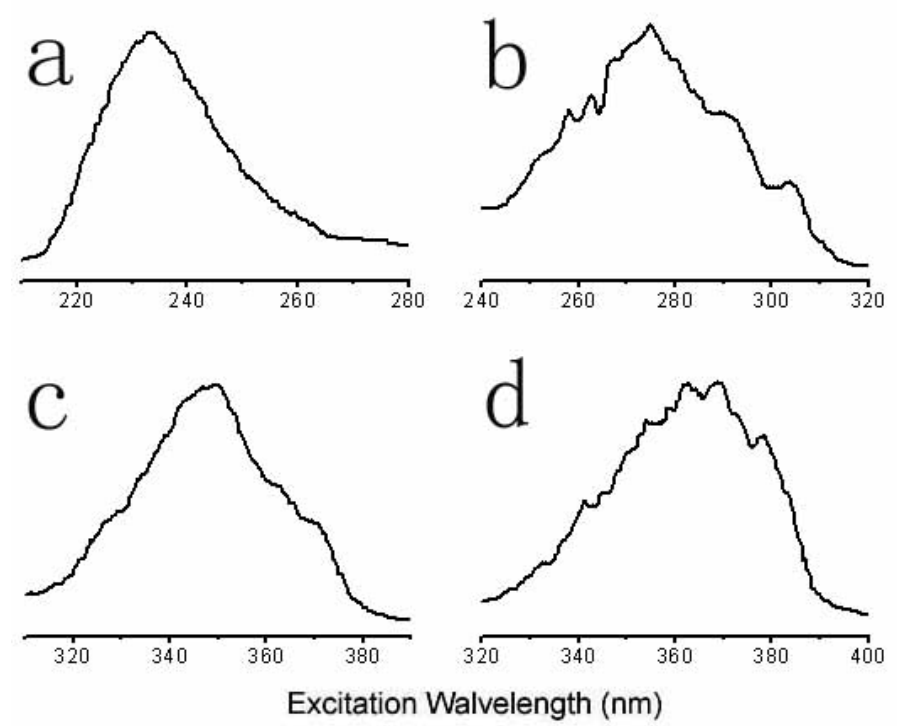

Figure S3 Excitation spectra of (a) 1, (b) $\sim 2$, (c) $\sim 3$, and (d) $\sim 4 \mathrm{~nm}$ diameter SiQDs for a, b, c, and d, respectively. SiQDs were prepared by using HPOM as the catalyst under current density less than 20 $\mathrm{mA} / \mathrm{cm}^{2}$.

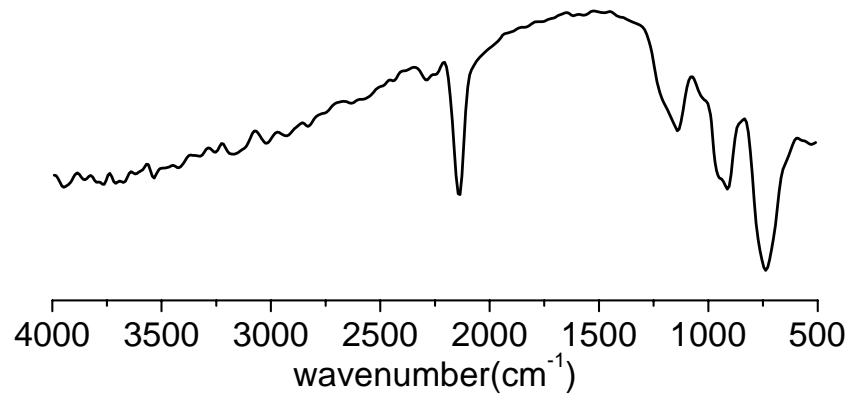

Figure S4 Typical IR spectrum of the as-prepared SiQDs using HPOM as the catalyst and current density less than $20 \mathrm{~mA} / \mathrm{cm}^{2}$.

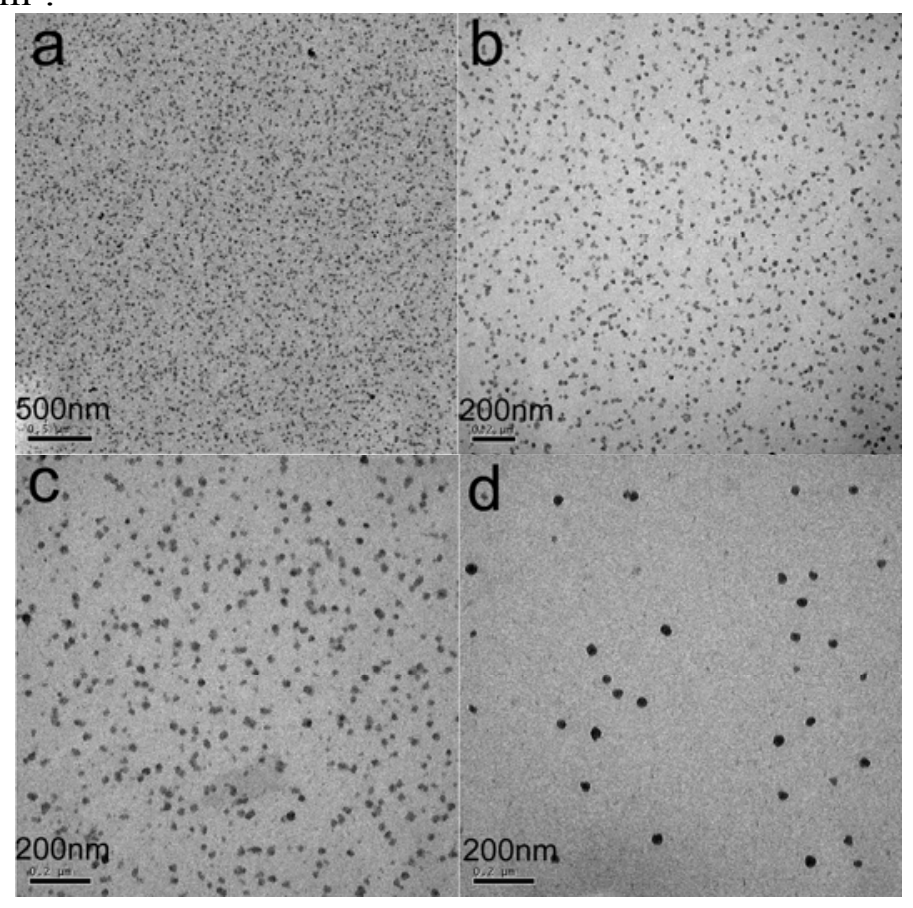

Figure S5 Typical TEM images (different magnification) of SiNPs with diameters $30-50 \mathrm{~nm}$. POM is the catalyst, and current density is less than $20 \mathrm{~mA} / \mathrm{cm}^{2}$. 


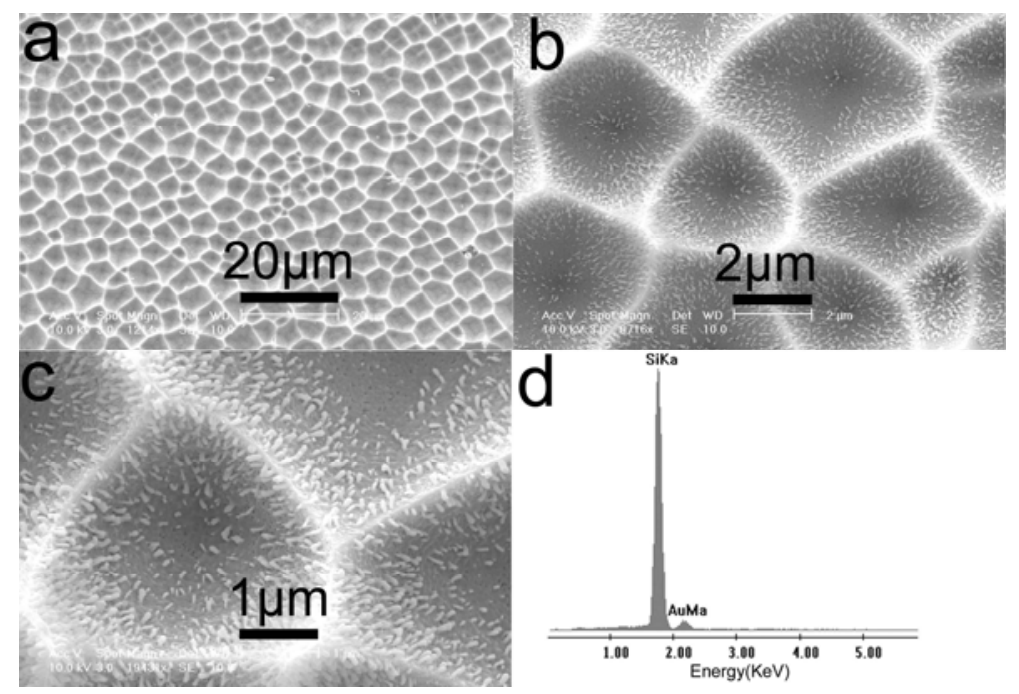

Figure S6 Typical SEM images (different magnification) of Si nanoparticles-in-micropore structures and the EDS spectrum. HPOM is the catalyst, and current density is increased to $20-50 \mathrm{~mA} / \mathrm{cm}^{2}$. Reaction time is about $1 \mathrm{~h}$.

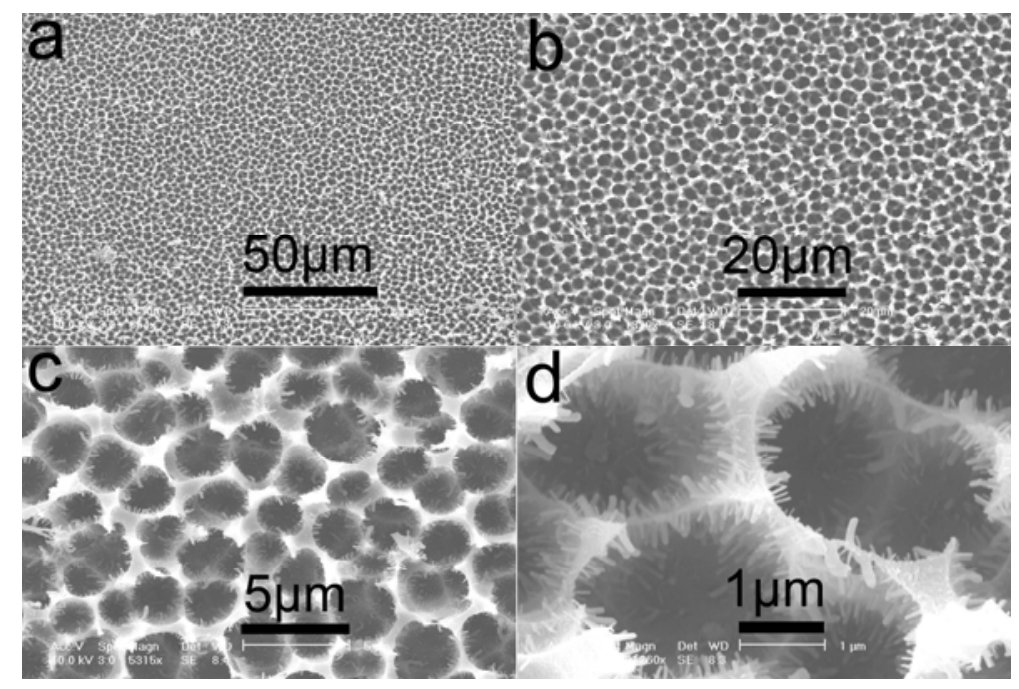

Figure S7 Typical SEM images (different magnification) of Si nanorods-in-micropore structures. HPOM is the catalyst, and current density is in the range of $20-50 \mathrm{~mA} / \mathrm{cm}^{2}$. Reaction time is about $4 \mathrm{~h}$.

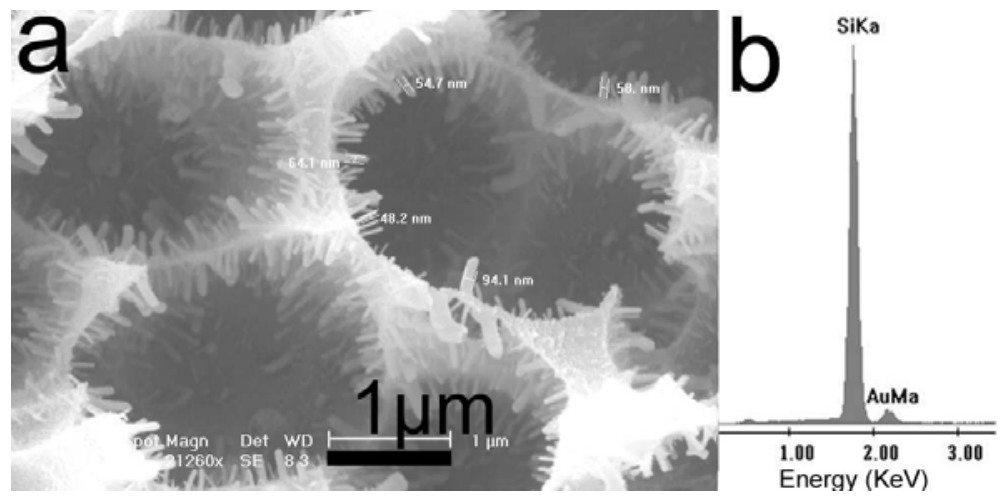

Figure S8 Typical SEM image (a) of Si nanorods-in-micropore structures and the EDS spectrum (b). HPOM is the catalyst, and current density is in the range of $20-50 \mathrm{~mA} / \mathrm{cm}^{2}$. Reaction time is about $4 \mathrm{~h}$. 


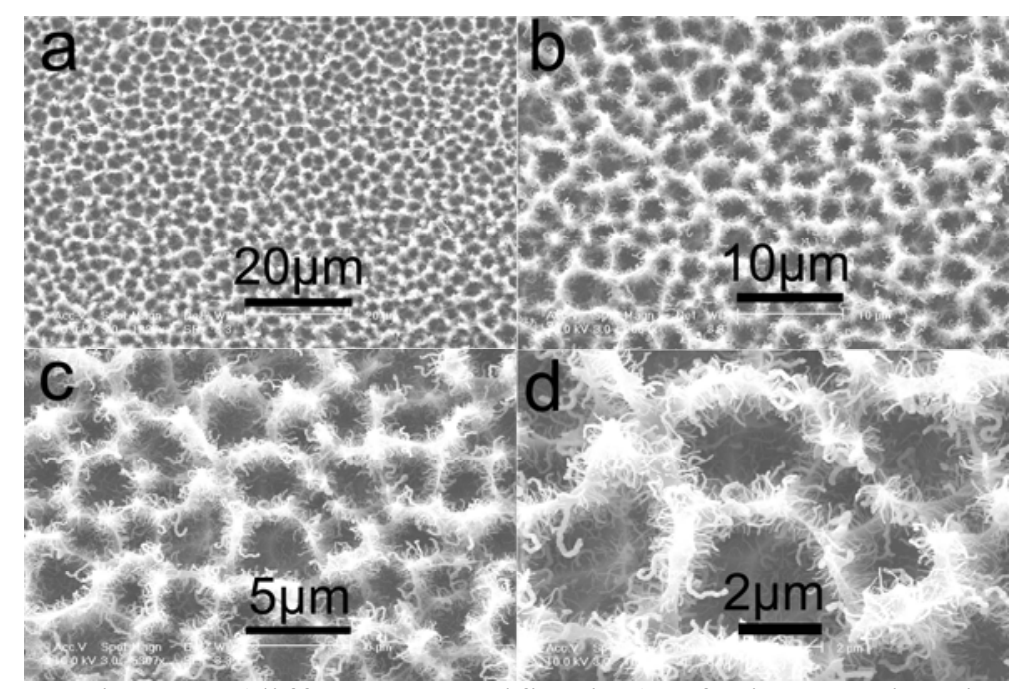

Figure S9 Typical SEM images (different magnification) of Si nanowires-in-micropore structures. HPOM is the catalyst, and current density is in the range of $20-50 \mathrm{~mA} / \mathrm{cm}^{2}$. Reaction time is about $7 \mathrm{~h}$.

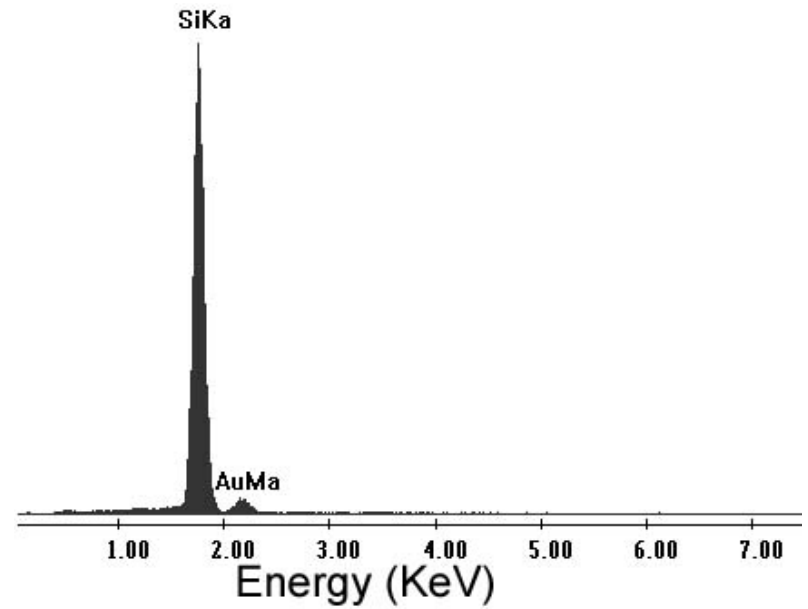

Figure S10 EDS spectrum of Si nanowires-in-micropore structures. HPOM is the catalyst, and current density is in the range of $20-50 \mathrm{~mA} / \mathrm{cm}^{2}$. Reaction time is about $4 \mathrm{~h}$.

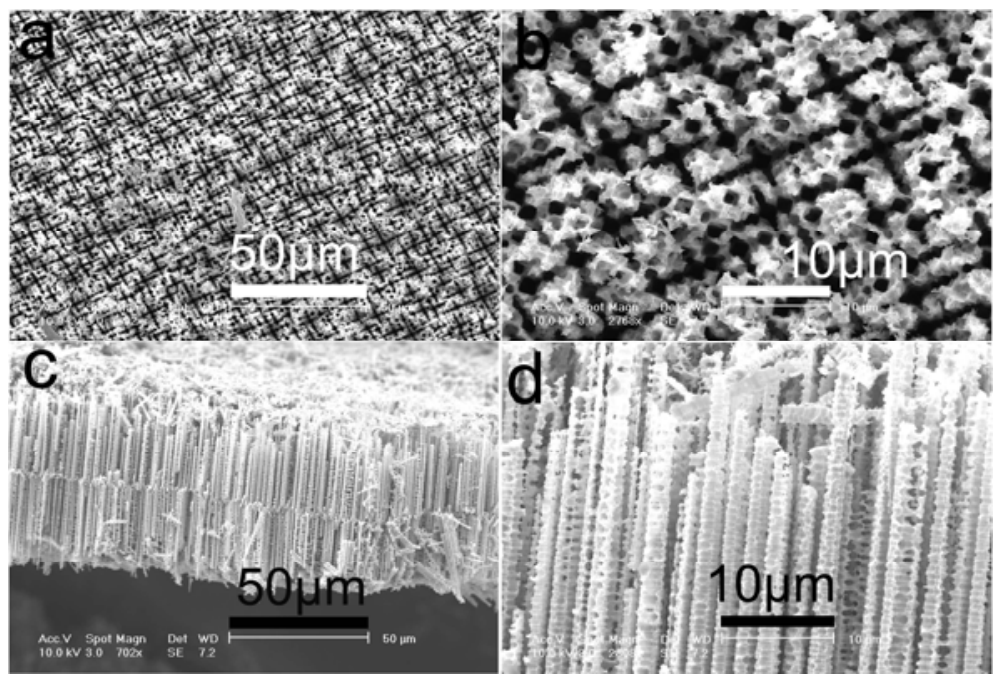

Figure S11 Typical SEM images (different magnification) of silicon pillars. $\mathrm{H}_{2} \mathrm{O}_{2}$ is the additive. Current density is in the range of $20-50 \mathrm{~mA} / \mathrm{cm}^{2}$. Reaction time is about $5 \mathrm{~h}$. 


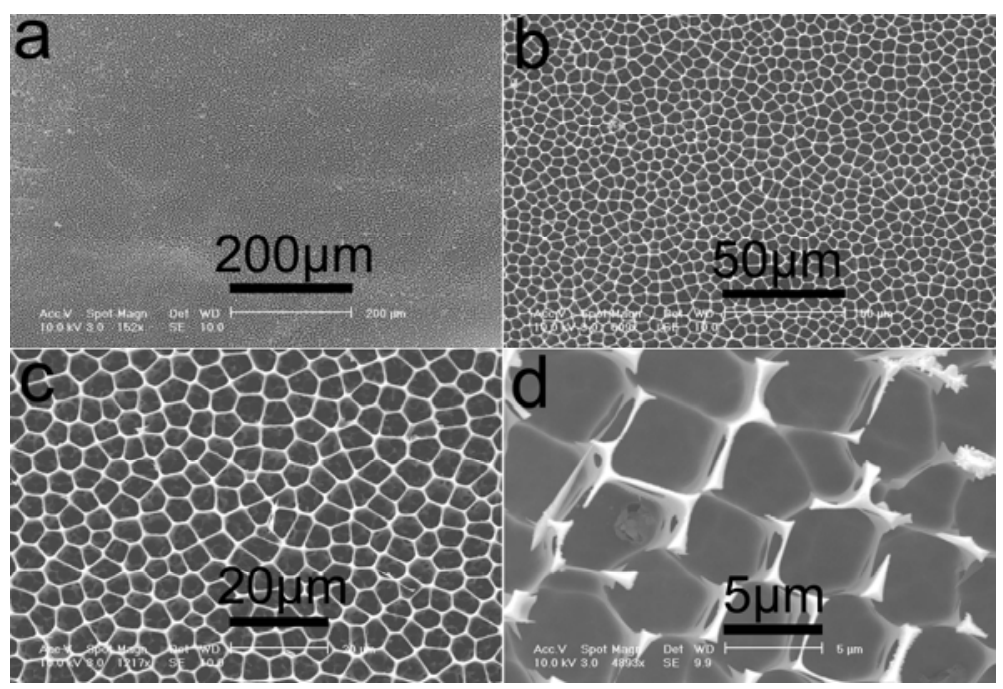

Figure S12 Typical SEM images (different magnification) of micro-sized holes silicon structures. $\mathrm{H}_{2} \mathrm{O}_{2}$ is the additive. Current density is in the range of $20-50 \mathrm{~mA} / \mathrm{cm}^{2}$. Reaction time is about $7 \mathrm{~h}$.

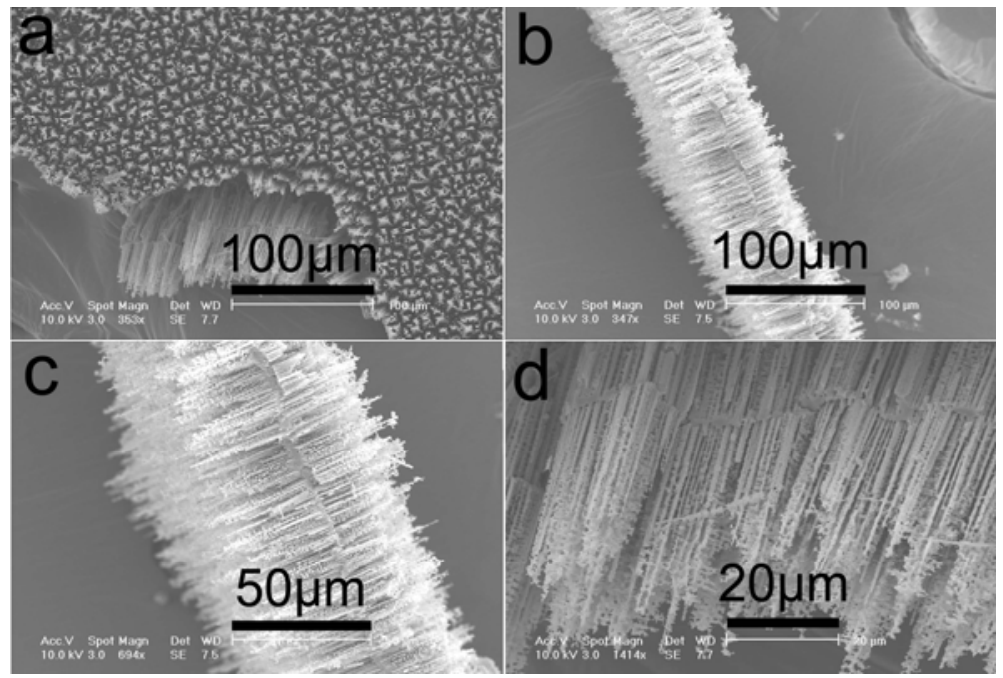

Figure S13 Typical SEM images (different magnification) of dendriform-like silicon structures. POM is the catalyst. Current density is over $50 \mathrm{~mA} / \mathrm{cm}^{2}$.

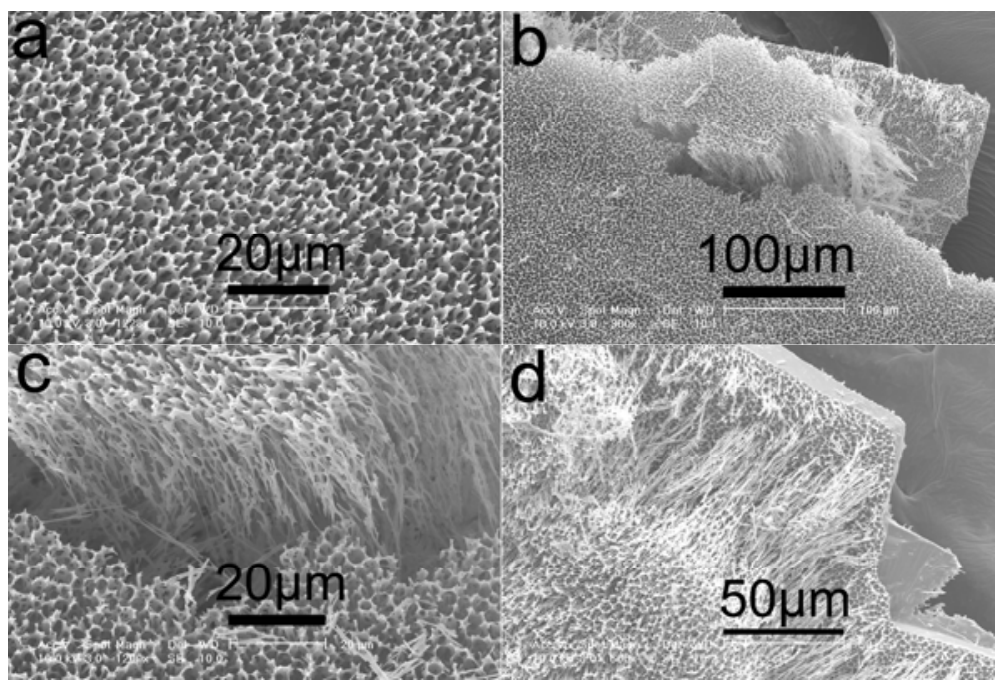


Figure S14 Typical SEM images (different magnification) of dendriform-like silicon structures. HPOM is the catalyst. Current density is over $50 \mathrm{~mA} / \mathrm{cm}^{2}$.

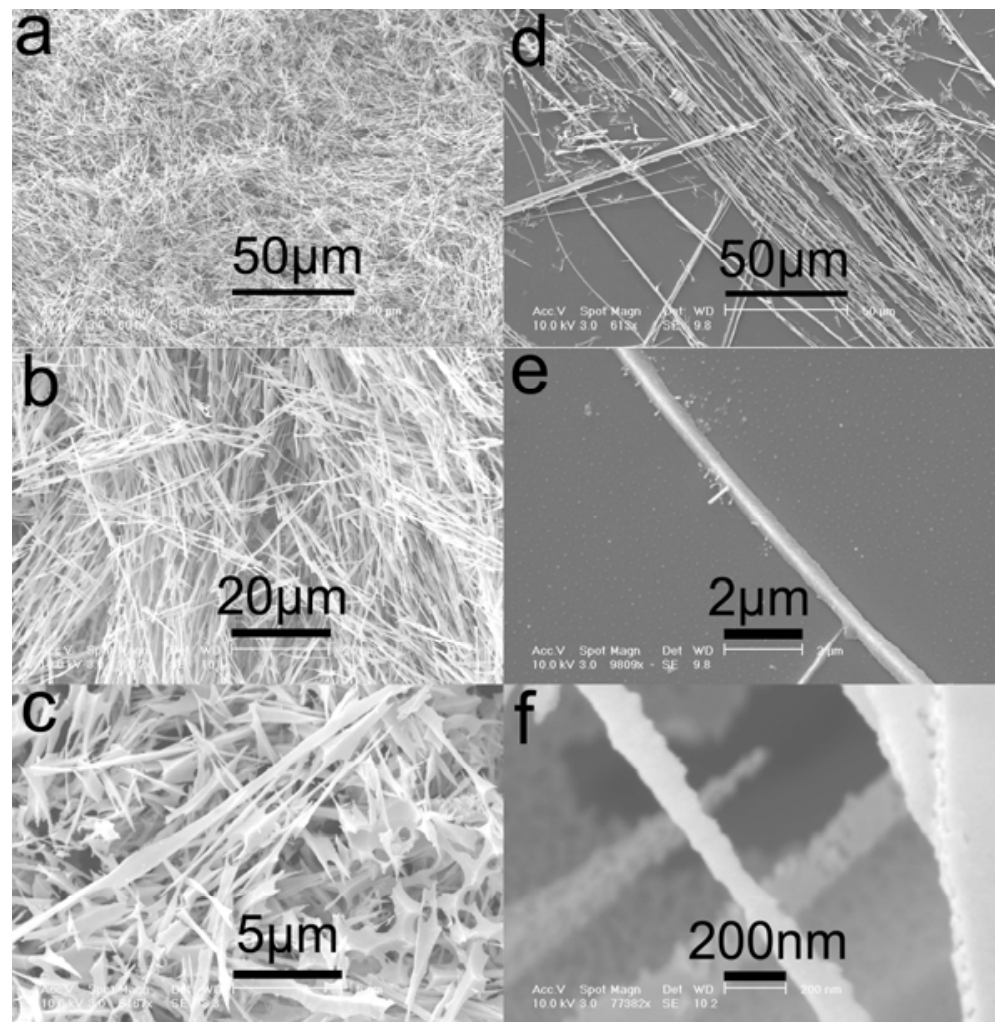

Figure S15 Typical SEM images of micro-sized wire-like silicon structures. $\mathrm{H}_{2} \mathrm{O}_{2}$ is the additive. Current density is over $50 \mathrm{~mA} / \mathrm{cm}^{2}$.

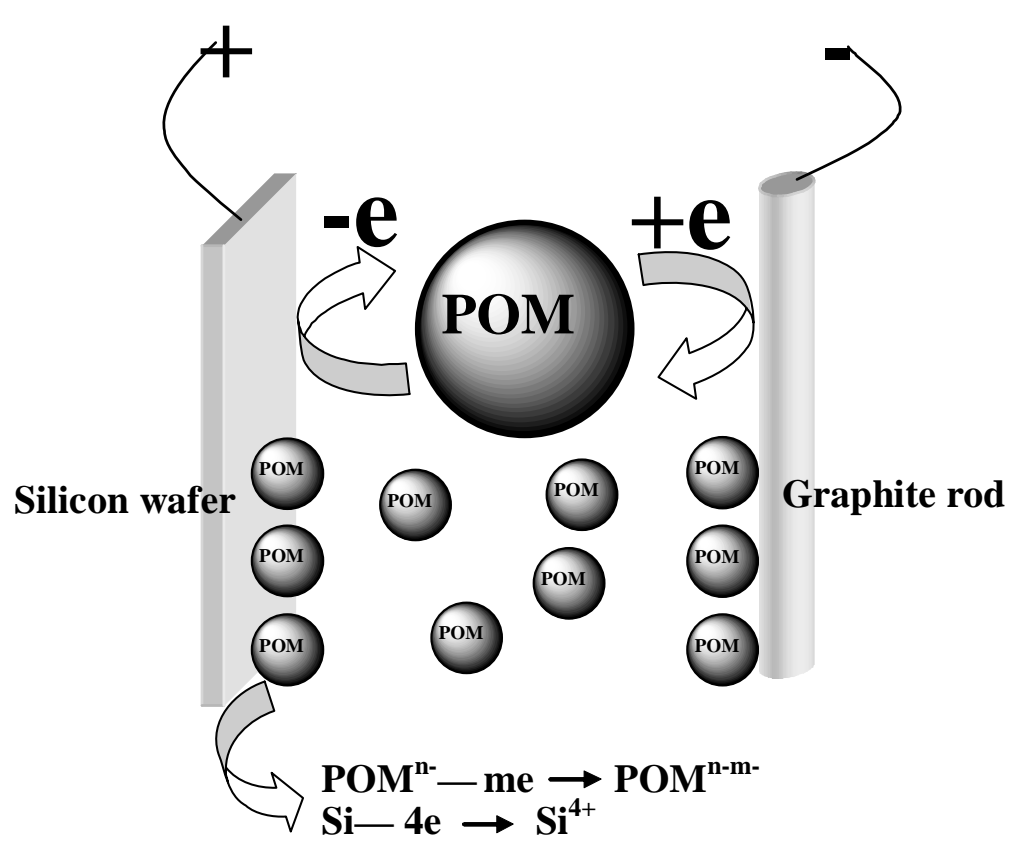

Figure S16. Schematics for the POMs-assisted electrochemical etching process. 